\title{
Commentary: Postoperative atrial fibrillation can last years? Oh snap!
}

\author{
Bobby Yanagawa, MD, PhD, ${ }^{a} \mathrm{Niv}$ Ad, $\mathrm{MD},{ }^{b}$ and Mario F. Gaudino, $\mathrm{MD}^{\mathrm{c}}$
}

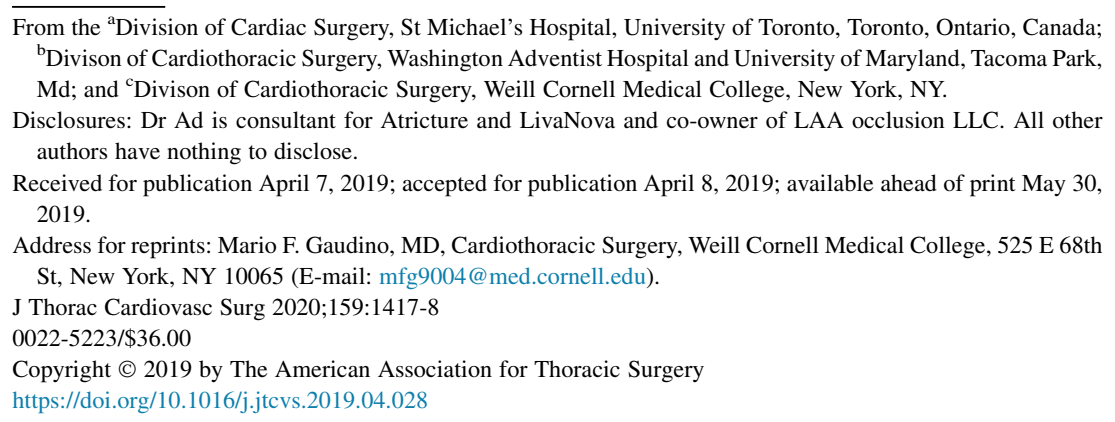

Despite improvements in perioperative and operative care, postoperative atrial fibrillation (POAF) is still found in approximately $15 \%$ to $60 \%$ of cardiac surgical patients. ${ }^{1}$ The potential clinical impact on patients is dependent on mainly their clinical status and can include heart failure, acute renal failure, stroke or thromboembolism, anticoagulation-related complications, and even death. This is particularly true because a significant proportion of patients with aortic stenosis have diastolic dysfunction and rely on the atrial contraction to maintain adequate cardiac output. More recently, it has been suggested that POAF is not just an acute phenomenon but rather that it may beget long-term risk of atrial fibrillation (AF). ${ }^{1}$ In this issue of the Journal, Axtell and associates ${ }^{2}$ present their findings evaluating the incidence of POAF in a retrospective study of 720 patients with no previous history of AF who underwent surgical aortic valve replacement (SAVR). Axtell and associates $^{2}$ report POAF in $46 \%$ of patients. Importantly, $25 \%$ (170 of 720) had "prolonged AF" (ie, AF detected by electrocardiography within 30 days and then again at another setting $>30$ days thereafter), and left atrial enlargement and old age were independent predictors. That larger atrial size is associated with prolonged POAF is not a new finding. The mechanism is not known but may be related to higher end-diastolic pressure and left atrial strain, as well as fibrous deposition and myocardial disarray. Advanced age is also a well-known risk factor for $\mathrm{AF}^{3}$ A potential mechanism is mitochondrial dysfunction leading to abnormal response to ischemic reperfusion injury and predisposing to $\mathrm{AF}^{4}$ The median duration of prolonged AF was 1.6 years. This was associated with a trend toward decrease in long-term survival, but it was not an independent risk factor for such.

The incidence of POAF after hospital discharge but within 30 days after coronary artery bypass grafting and valve surgery has been reported to be in the range of $2 \%$ to $8 \%{ }^{5}$ Importantly, the method of $\mathrm{AF}$ detection affects the

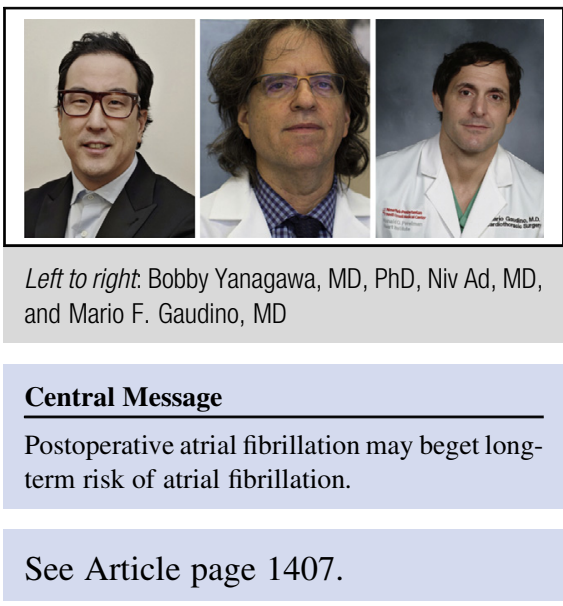

incidence. In the study of Axtell and associates, ${ }^{2} 25 \%$ of patients had new, prolonged AF, as detected by in-office 12-lead electrocardiography. This likely captured most patients with persistent AF, but it may have missed at least some patients with paroxysmal AF. ${ }^{5}$ Ultimately, more sensitive rhythm monitoring modalities are needed to determine the true AF burden in patients after discharge. In this regard, the Post-Surgical Enhanced Monitoring for Cardiac Arrhythmias and Atrial Fibrillation trial (SEARCH-AF; ClinicalTrials.gov identifier NCT02793895) is an ongoing multicenter randomized clinical trial that compares the use of a wearable, adhesive patch-based cardiac rhythm monitor against the usual care standard for the detection of POAF during the subacute period. This trial will provide information on the subacute burden of POAF, but the cost and practicality of routine intense rhythm monitoring may not make it usable outside a trial. Regarding the incidence of POAF, it is important to point out that there is a baseline risk of $\mathrm{AF}$ that increases with age: $2.3 \%$ in people older than 40 years and $5.9 \%$ in those older than 65 years. Also, although these patients had no previous AF, this assessment is based on clinical history alone, because few patients undergo preoperative rhythm monitoring.

Axtell and colleagues ${ }^{2}$ suggest prospective surgical trials to assess the effectiveness of surgical ablation-Cox-maze procedure or pulmonary vein isolation (PVI) — or left atrial appendage (LAA) isolation or amputation for reduction of incidence of POAF and for stroke prevention, respectively. We agree that targeted efforts to reduce the burden of POAF are needed. Prophylactic surgical ablation for POAF, however, has never been proved effective. The rationale for 
PVI to treat paroxysmal AF is based on the pioneering work of Haïssaguerre and associates, ${ }^{6}$ which showed that more than $90 \%$ of ectopic foci mapped to the pulmonary veins. On the other hand, the mechanism of POAF is likely related to some combination of local proinflammatory milieu, increased adrenergic tone, and fluid and electrolyte imbalance. As a proof of concept, Kiaii and colleagues ${ }^{7}$ performed a small, randomized trial of PVI versus no PVI in 175 patients undergoing coronary artery bypass grafting. In this pilot trial, there was no signal to suggest that PVI may lower the incidence of POAF (37\% vs $36 \%$; $P=.8$ ). Furthermore, fewer than half the patients with AF coming for surgery have some concomitant surgical ablation in the Society of Thoracic Surgeons, database so it is unlikely that surgeons would open both atria to complete the full biatrial lesion set for a potential reduction in $\mathrm{POAF}^{8}$ Finally, success or failure of surgical ablation is assessed only after the 3 -month blanking period. The rationale is to allow some period of time after radiofrequency or cryoablation to allow the scar formation that is ultimately responsible for conduction block. In our experience, $20 \%$ to $40 \%$ of patients are in AF immediately after a Coxmaze IV procedure. Although the precise duration of time needed is unknown, and 3 months is admittedly somewhat arbitrary, it is generally accepted that some time needs to pass to allow the tissue to scar. From a timing standpoint, it is therefore unlikely that fresh ablation lesions can be used to treat POAF.

Regarding LAA isolation or amputation for stroke prevention, there have been mixed results. No data currently exist to support routine LAA closure at the time of cardiac surgery. The Left Atrial Appendage Occlusion Study III is an ongoing trial randomly assigning 4812 patients with AF undergoing cardiac surgery to receive LAA occlusion or usual care (LAAOS III, ClinicalTrials.gov identifier NCT01561651). The primary outcome is stroke or thromboembolism at 4 years. The results of this trial will provide valuable information on the efficacy of LAA isolation or amputation for stroke prevention in an enriched surgical population.

As a final note, the recognition of subclinical, hypoattenuated leaflet thickening and reduced leaflet motion after bioprosthetic aortic valve replacement has raised much discussion of the role of short-term anticoagulation. ${ }^{9}$ If indeed almost half of all patients have transient POAF, and a quarter have prolonged POAF, then routine short-term anticoagulation may protect patients from both subclinical valve thrombosis and AF-related thromboembolism.

\section{References}

1. Ha AC, Mazer CD, Verma S, Yanagawa B, Verma A. Management of postoperative atrial fibrillation after cardiac surgery. Curr Opin Cardiol. 2016; 31:183-90.

2. Axtell AL, Moonsamy P, Melnitchouk S, Tolis G, Jassar AS, D'Alessandro DA, et al. Preoperative predictors of new onset prolonged atrial fibrillation after surgical aortic valve replacement. J Thorac Cardiovasc Surg. 2020;159:1407-14.

3. Feinberg WM, Blackshear JL, Laupacis A, Kronmal R, Hart RG. Prevalence, age distribution, and gender of patients with atrial fibrillation. Analysis and implications. Arch Intern Med. 1995;155:469-73.

4. Ad N, Schneider A, Khaliulin I, Borman JB, Schwalb H. Impaired mitochondrial response to simulated ischemic injury as a predictor of the development of atrial fibrillation after cardiac surgery: in vitro study in human myocardium. $J$ Thorac Cardiovasc Surg. 2005;129:41-5.

5. Ha ACT, Verma A, Mazer CD, Yanagawa B, Verma S. The more you look, the more you find: atrial fibrillation-nowhere to hide. Curr Opin Cardiol. 2019;34: 140-6.

6. Haïssaguerre M, Jaïs P, Shah DC, Takahashi A, Hocini M, Quiniou G, et al. Spontaneous initiation of atrial fibrillation by ectopic beats originating in the pulmonary veins. N Engl J Med. 1998;339:659-66.

7. Kiaii B, Fox S, Chase L, Fernandes M, Stitt LW, Guo R, et al. Postoperative atrial fibrillation is not pulmonary vein dependent: results from a randomized trial. Heart Rhythm. 2015;12:699-705.

8. Badhwar V, Rankin JS, Ad N, Grau-Sepulveda M, Damiano RJ, Gillinov AM, et al. Surgical ablation of atrial fibrillation in the United States: trends and propensity matched outcomes. Ann Thorac Surg. 2017;104:493-500.

9. Makkar RR, Fontana G, Jilaihawi H, Chakravarty T, Kofoed KF, de Backer O, et al. Possible subclinical leaflet thrombosis in bioprosthetic aortic valves. $N$ Engl J Med. 2015;373:2015-24. 